\title{
CUIDADO COM A GESTANTE OBESA: COMO PREVENIR COMPLICAÇÕES?
}

\section{Categoria Clínico}

INSTITUIÇÃO: Centro Universitário São Camilo.

AUTORES: Molina $\mathrm{CA}^{1}$;

Rua Acuruá, 97 Vila Romana - São Paulo (SP)- 05053-000; (11) 966302806; e-mail: cmol.assis@gmail.com;

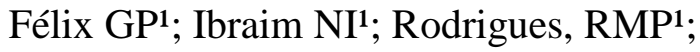

ORIENTADORES: Pereira MM²; Vilarino FL.

Discentes da Faculdade de Medicina do Centro Universitário São Camilo ${ }^{1}$; Docentes da Faculdade de Medicina do Centro Universitário São Camilo 
CUIDADO COM A GESTANTE OBESA: COMO PREVENIR COMPLICAÇÕES?

Categoria Clínico

DESCRITORES: “OBESITY”; "PREGNANCY COMPLICATIONS”; "HEALTH CARE” 


\section{RESUMO}

INTRODUÇÃO: Sobrepeso e obesidade representam um cenário complicado no período gestacional e no momento do trabalho de parto. Mulheres com IMC $>28 \mathrm{~kg} / \mathrm{m}^{2}$ durante a gestação apresentam maiores riscos, como diabetes gestacional, hipertensão, além de complicações para o neonato como macrossomia e hipoglicemia. Nesse âmbito, a adesão a um estilo de vida mais saudável, tanto antes quanto durante a gestação, evidencia-se como um potencial fator de prevenção.

METODOLOGIA: Realizada pesquisa no banco de dados PubMed, utilizando-se os descritores: “obesity", "pregnancy complications" e "healthcare" e os critérios de inclusão "publicação nos últimos 5 anos" e "idioma (português, inglês ou espanhol)". Um total de 426 artigos foram encontrados e 27 foram selecionados de acordo com os critérios de exclusão "estudos secundários" e "não adequação ao objetivo".

OBJETIVOS: Avaliar a literatura acerca das particularidades na gestação de mulheres obesas ou com sobrepeso, buscando medidas de prevenção para complicações neonatais e maternas.

RESULTADOS: Os estudos analisados levaram em consideração o peso prévio ao início da gestação e identificaram mulheres com $30 \mathrm{~kg} / \mathrm{m}^{2}<\mathrm{IMC}>28 \mathrm{~kg} / 2$ como gestantes com sobrepeso e aquelas com IMC $>30 \mathrm{~kg} / \mathrm{m}^{2}$ como gestantes obesas. Nessas mulheres, observou-se maior risco de desenvolver diabetes gestacional, hipertensão e maior ocorrência de eventos cardiovasculares durante e após a gestação. Diante disso, a adoção de rotinas de exercícios (pelo menos 30 minutos, 3 vezes por semana) e de uma alimentação controlada impactou no ganho de peso médio durante a gestação (diferença média de $-0,92 \mathrm{~kg}$ ) e no peso médio ao nascer dos neonatos (diferença média de - $104 \mathrm{~g}$ ). As gestantes submetidas a rotina regular de exercícios demonstraram uma incidência 18,6\% menor de diabetes gestacional e uma redução de 7,73mmHg na pressão arterial sistólica. Ademais, os estudos indicam que essas intervenções podem favorecer o momento do trabalho de parto, reduzindo a ocorrência de distócias de ombros e cesáreas de emergência.

CONCLUSÃO: A literatura analisada evidencia a obesidade como um importante agravante e fator de risco para complicações gestacionais e neonatais. Logo, é evidente a necessidade de um manejo individualizado nesses casos, visando intervenções precoces como a orientação de uma alimentação balanceada e a adoção de uma rotina regular de exercícios.

DESCRITORES: “obesity”; "pregnancy complications"; "health care” 


\section{CUIDADO COM A GESTANTE OBESA: COMO PREVENIR COMPLICAÇÕES?}

\section{INTRODUÇÃO}

Segundo a Organização Mundial de Saúde, o excesso de peso é definido como a acumulação de gordura anormal ou excessiva que pode prejudicar a saúde. ${ }^{\left({ }_{1}\right)}$ Esse excesso de peso pode ser avaliado usando o índice de massa corporal (IMC), sendo que, um IMC maior ou igual a $30 \mathrm{~kg} / \mathrm{m} 2$ é considerado obesidade. ${ }^{(2)}$

A obesidade está associada a um grande número de complicações, tais como, diabetes mellitus tipo 2, dislipidemia, hipertensão, doença cardiovascular, entre outros, estando relacionada a um aumento da mortalidade, na sua maioria associada a doença cardiovascular. ${ }^{\left({ }^{1}\right)}$

A prevalência da obesidade mundial vem aumentando ao longo das últimas décadas, se tornando um grave problema de saúde pública, sua epidemia se deve a vários fatores, como, por exemplo, o avanço dos meios de transporte, o aumento da disponibilidade de equipamentos que facilitam o desempenho de atividades de vida diária, sedentarismo e o fácil acesso a comida (sendo os alimentos ricos em gordura e açúcar geralmente os mais baratos). ${ }^{(3)}$

A gestação pode agir como desencadeante da obesidade assim como agravante dela, principalmente quando existir um excesso de peso pré-existente. ${ }^{(3)}$ Sabe-se que a gravidez em mulheres com obesidade possui implicações a longo prazo tanto para a saúde materna quanto a infantil, se tornando assim, um importante problema de saúde pública. ${ }^{\left({ }_{2}\right)}$ Além disso, a obesidade gestacional denota maior risco de abortamento espontâneo, malformações congênitas, diabetes gestacional, doença hipertensiva da gravidez, macrossomia, baixo peso de nascimento e cesárea, entre outros. ${ }^{(1)}$

Para diminuir a prevalência de obesidade materna e assim diminuir suas complicações e riscos, diversas estratégias têm sido aplicadas, tais como, perda de peso prévia à gravidez, mudanças no estilo de vida com adequação de hábitos alimentares e de rotina de exercício físico. ${ }^{\left({ }^{1}\right)}$ Além disso, acompanhamento multidisciplinar e suporte psicológico são necessários nesse período para estimular a perda de peso e hábitos mais saudáveis, visando uma dieta rica em cálcio, vitamina $\mathrm{D}$, ferro e folato. $(2)(4)$

\section{OBJETIVOS}

\subsection{OBJETIVO GERAL}

Avaliar a literatura acerca das particularidades na gestação de mulheres obesas ou com sobrepeso, buscando medidas de prevenção para complicações neonatais e maternas.

\subsection{OBJETIVOS ESPECÍFICOS}

Identificar as complicações maternas mais comuns na gestação de mulheres obesas. 
Identificar as complicações fetais mais comuns na gestação me mulheres obesas.

Avaliar quais as medidas de prevenção de complicações na gestação de mulheres obesas apresentam maior nível de evidência e eficácia.

\section{METODOLOGIA}

O estudo foi desenvolvido por meio de uma revisão da literatura utilizando-se a base de dados PubMed. Com os descritores "obesity", "pregnancy complications" e "healthcare" foram encontrados um total de 426 estudos e selecionados 27. Os critérios de inclusão adotados foram "publicação nos últimos 5 anos" e "idioma" (inglês, português e espanhol). Os critérios de exclusão foram "estudos secundários" e "não adequação ao objetivo". (Fig.1)

\section{PESQUISA NA BASE DE DADOS PubMED}

“obesity”AND "pregnancy complications" AND "healthcare"

\section{CRITÉRIOS DE INCLUSÃO}

- Ano de publicação

2014 a 2018

- Idioma

Português, inglês e espanhol

\section{ESTUDOS ENCONTRADOS}

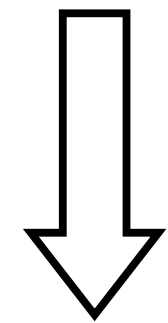

\section{CRITÉRIOS DE EXCLUSÃO}

- Estudos secundários

- Não adequação ao objetivo

\section{ESTUDOS SELECIONADOS}

Fig.1. Fluxograma de metodologia. 


\section{RESULTADOS}

Todos os estudos analisados levaram em consideração o peso prévio ao início da gestação e identificaram mulheres com $30 \mathrm{~kg} / \mathrm{m}^{2}<\mathrm{IMC}>28 \mathrm{~kg} / 2$ como gestantes com sobrepeso e aquelas com IMC $>30 \mathrm{~kg} / \mathrm{m}^{2}$ como gestantes obesas.

Mulheres com excesso de peso ou obesas durante a gravidez correm maior risco de inúmeras complicações, incluindo diabetes gestacional, hipertensão e pré-eclâmpsia, necessidade de cesariana e crianças com alto peso ao nascer..$^{(5)}$ Essas mulheres também apresentam maior risco de desenvolver diabetes, hipertensão e doença cardiovascular na vida adulta, condições que implicam em um risco aumentado de morte prematura, em grande parte relacionado a eventos cardiovasculares maiores. ${ }^{(5)}$ Além disso, destacam-se complicações fetais: crianças nascidas de mulheres com sobrepeso ou obesas têm maior probabilidade de parto prematuro e suas consequencias. ${ }^{(5)}$

Boudet-Berquier et al, analisou as características sociodemográficas associadas ao sobrepeso e obesidade gestacional. Esse estudo identificou que a paridade modula a relação entre os níveis de IMC e inúmeras características das gestantes. Entre mulheres primíparas, obesidade foi associada a uma idade materna de 25-29 anose com diabetes mellitus gestacional ecomplicações hipertensivas durante a gravidez. ${ }^{(6)}$ Entre as multíparas, a obesidade materna foi associada à ausência de frequência nas aulas pré-natais, a diabetes mellitus gestacional, complicações hipertensivas, excesso de ganho de peso durante a gestação, parto cesáreo e pesoao nascer $\geq 4 \mathrm{~kg} .{ }^{\left({ }^{6}\right)}$

Washington et al, avaliou o atendimento ante-natal e pré-natal oferecido as mulheres, questionando possíveis divergências nas orientações e forma de comunicação utilizada nos casos de sobrepeso e obesidade.Em comparação com mulheres com peso normal, os profissionais fizeram menos perguntas (IRR 0,66, IC 95\% 0,44-0,99, p =0,04) e deram menos informações sobre estilo de vida (IRR 0,51, IC 95\% 0,32-0,82, p = 0,01) a mulheres com sobrepeso e obesidade. ${ }^{\left({ }^{7}\right.}$ ) Os profissionais de saúde usaram menos aprovação (IRR 0,68, IC 95\% 0,51-0,91, p = 0,01) e declarações de preocupação (IRR 0,68, IC 95\% 0,53-0,86, p = 0,002) ao cuidar de mulheres com sobrepeso e menos declarações de acolhimento para mulheres com obesidade (IRR 0,40, IC 95\% 0,19-0,84 p = 0,02)..$\left.^{7}\right)$ Nesse contexto, o autor sugere que intervenções para aumentar o uso de comunicação centrada no paciente - especialmente para mulheres com sobrepeso e obesidade - podem melhorar a qualidade do atendimento pré-natal. ${ }^{(7)}$

Em relação às possíveis intervenções eficazes na alteração desse cenário, Dencker et al avaliou fatores que possam interferir na adesão a mudanças no estilo de vida. O autor conclui, a partir de entrevistas com as gestantes que, para apoiar mulheres com IMC $\geq 30 \mathrm{~kg} / \mathrm{m}$ (2) a fazer mudanças 
no estilo de vida saudável e limitar o ganho de peso durante a gravidez, os provedores de serviços de saúde pré-natais devem (1) abordar o peso das mulheres de maneira não julgadora usando o IMC e fornecer informações precisas e apropriadas sobre os benefícios do ganho de peso gestacional limitado; (2) apoiar a mulher nos seus próprios termos numa relação de colaboração com a parteira; (3) trabalhar em parceria para dar à mulher as ferramentas para auto-gerenciar atividades saudáveis e (4) dar apoio pessoal contínuo e monitoramento para manter uma alimentação saudável e hábitos regulares de atividade física após o parto envolvendo também o parceiro e a família. ${ }^{\left({ }^{8}\right)}$

Todos os estudos parecem concordar que a prevenção é a mais importante intervenção necessária para essas mulheres e deve se pautar em três pilares: mudança de hábitos, exercícios físicos (pelo menos 30 minutos de caminhadas, 3 vezes por semana) e acompanhamento pré-natal adequado.

Garnaes et al, demonstrou, em mulheres submetidas a atividades físicas, ganho de peso gestacional médio do início ao parto de 10,5 kg no grupo de exercício e 9,2 kg no grupo de controle, com uma diferença média de $\left.0,92 \mathrm{~kg}(95 \% \mathrm{CI}-1,35,3,18 ; \mathrm{p}=0,43) .{ }^{9}{ }^{9}\right)$ Entre os 30 desfechos secundários no final da gravidez, uma aparente redução foi registrada na incidência de diabetes mellitus gestacional no grupo de exercício ( 2 casos; $6,1 \%$ ) comparado ao grupo controle (9 casos; $27,3 \%$ ), com um odds ratio de 0,1 (IC 95\% 0,02, 0,95; $\mathrm{p}=0,04) .{ }^{\left({ }^{9}\right)}$ A pressão arterial sistólica foi significativamente menor no grupo de exercício (média de 120,4 mm Hg) em comparação com o grupo controle (média de 128,1 mm Hg), com uma diferença média de -7,73 mm Hg (IC 95\% -13,23, $\left.-2,22 ; \mathrm{p}=0,006) \cdot \cdot^{9}\right)$

Seneviratne et al, também avaliou a eficácia da adoção de exercícios físicos no período antenatal. Foi observado que o condicionamento aeróbico melhorou no grupo de intervenção em comparação com os controles (melhora de 48,0 segundos no tempo de teste para frequência cardíaca alvo; $\mathrm{P}=0,019) .{ }^{\left({ }^{0}\right)}$ Análises de sensibilidade demonstraram que maior adesão foi associada à melhora da condição física (aumento do tempo de teste $(\mathrm{P}=0,002)$, maior VO2 pico $(\mathrm{P}=0,015)$ e menor frequência cardíaca de repouso $(P=0,014)$, redução da adiposidade pós-natal $\mathrm{P}=0,007)$ e índice de massa corporal $(\mathrm{P}=0,035))$ e melhor qualidade de vida física $(\mathrm{P}=0,034) \cdot .^{\left(1^{0}\right)}$

\section{CONCLUSÕES}

Toda a literatura analisada apresenta o sobrepeso e a obesidade durante a gestação como um importante fator de risco para complicações maternas e fetais. Sendo assim, é de extrema importância uma intervenção precoce, de preferência antes da gestação, para que a mulher tenha uma alimentação mais saudável e implementar a prática regular de exercícios físicos moderados a fim de diminuir o peso. 
Os estudos apontam a gestação como um período oportuno para intervenções, sendo considerado um momento de aprendizado no qual a mulher encontra-se interessada em promover uma mudança de comportamento, principalmente pela sua principal motivação, a saúde da criança. Por conta disso, é recomendado o acompanhamento com uma equipe multidisciplinar, pelo fato de sentirem-se desamparadas.

É importante que esse acompanhamento seja isento de qualquer tipo de julgamento, sendo abordado com sensibilidade e respeito, de forma que a mulher se sinta acolhida, fornecendo informações sobre os riscos da obesidade, tanto para ela quanto para a criança, orientação individualizada quanto ao seu plano alimentar e a prática de atividades físicas, realizando esse acompanhamento inclusive no puerpério e decorrer da vida.

\section{REFERÊNCIAS}

(1) Silva, Catarina Maria Miranda da. (2013).Obesidadee gravidez - Consequências e abordagem clínica da gravidaobesa.Curso de Medicina, Universidade de Coimbra, Término de conclusão de curso, f 38 .

(2) Dolin, Cara D., Kominiarek, Michelle A. (2018). Pregnancy in Women with Obesity. Obstetrics And Gynecology Clinics Of North America, 45 (2), pp.217-232

(3) Mattar, Rosiane et al. (2009). Obesidade e gravidez. Revista Brasileira de Ginecologia e Obstetricia, pp.107-110

(4) Dorise, Bonnie et al. (2018). A low intensity dietary intervention for reducing excessive gestational weight gain in an overweight and obese pregnant cohort. Eating And Weight Disorders - Studies On Anorexia, Bulimia And Obesity. Springer Nature America

(5) Moran, L., Fraser, L., Sundernathan, T., Deussen, A., Louise, J., Yelland, L., Grivell, R., Macpherson, A., Gillman, M., Robinson, J., Owens, J. and Dodd, J. (2017). The effect of an antenatal lifestyle intervention in overweight and obese women on circulating cardiometabolic and inflammatory biomarkers: secondary analyses from the LIMIT randomised trial. BMC Medicine, 15(1).

(6) Boudet-Berquier, J., Salanave, B., Desenclos, J. and Castetbon, K. (2017). Sociodemographic factors and pregnancy outcomes associated with prepregnancy obesity: effect modification of parity in the nationwide Epifane birth-cohort. BMC Pregnancy and Childbirth, 17(1).

(7) Washington Cole, K., Gudzune, K., Bleich, S., Cheskin, L., Bennett, W., Cooper, L. and Roter, D. (2017). Providing prenatal care to pregnant women with overweight or obesity: Differences in provider communication and ratings of the patient-provider relationship by patient body weight. Patient Education and Counseling, 100(6), pp.1103-1110. 
(8) Dencker, A., Premberg, Å., Olander, E., McCourt, C., Haby, K., Dencker, S., Glantz, A. and Berg, M. (2016). Adopting a healthy lifestyle when pregnant and obese - an interview study three years after childbirth. BMC Pregnancy and Childbirth, 16(1).

(9) Garnæs, K., Mørkved, S., Salvesen, Ø. and Moholdt, T. (2016). Exercise Training and Weight Gain in Obese Pregnant Women: A Randomized Controlled Trial (ETIP Trial). PLOS Medicine, 13(7), p.e1002079.

(10) Seneviratne, S., Parry, G., McCowan, L., Ekeroma, A., Jiang, Y., Gusso, S., Peres, G., Rodrigues, R., Craigie, S., Cutfield, W. and Hofman, P. (2014). Antenatal exercise in overweight and obese women and its effects on offspring and maternal health: design and rationale of the IMPROVE (Improving Maternal and Progeny Obesity Via Exercise) randomised controlled trial. BMC Pregnancy and Childbirth, 14(1).

(11) Atkinson, L., Olander, E. and French, D. (2015). Acceptability of a Weight Management Intervention for Pregnant and Postpartum Women with BMI $\geq 30 \mathrm{~kg} / \mathrm{m} 2$ : A Qualitative Evaluation of an Individualized, Home-Based Service. Maternal and Child Health Journal, 20(1), pp.88-96.

(12) Bruno, R., Petrella, E., Bertarini, V., Pedrielli, G., Neri, I. and Facchinetti, F. (2016). Adherence to a lifestyle programme in overweight/obese pregnant women and effect on gestational diabetes mellitus: a randomized controlled trial. Maternal \& Child Nutrition, 13(3), p.e12333.

(13) DeJoy, S., Bittner, K. and Mandel, D. (2016). A Qualitative Study of the Maternity Care Experiences of Women with Obesity: "More than Just a Number on the Scale". Journal of Midwifery \& Women's Health, 61(2), pp.217-223.

(14) Dinsdale, S., Branch, K., Cook, L. and Shucksmith, J. (2016). “As soon as you've had the baby that's it..." a qualitative study of 24 postnatal women on their experience of maternal obesity care pathways. BMC Public Health, 16(1).

(15) Dodd, J., Newman, A., Moran, L., Deussen, A., Grivell, R., Yelland, L., Crowther, C., McPhee, A., Wittert, G., Owens, J., Turnbull, D. and Robinson, J. (2015). The effect of antenatal dietary and lifestyle advice for women who are overweight or obese on emotional well-being: the LIMIT randomized trial. Acta Obstetricia et Gynecologica Scandinavica, 95(3), pp.309-318.

(16) Fieril, D., Olsén, P., Glantz, D. and Premberg, D. (2017). Experiences of a lifestyle intervention in obese pregnant women - A qualitative study. Midwifery, 44, pp.1-6.

(17) Heslehurst, N., Dinsdale, S., Brandon, H., Johnston, C., Summerbell, C. and Rankin, J. (2017). Lived experiences of routine antenatal dietetic services among women with obesity: A qualitative phenomenological study. Midwifery, 49, pp.47-53. 

Gestational Diabetes Mellitus. Midwifery, 49, pp.79-86.

(19) Knight-Agarwal, C., Williams, L., Davis, D., Davey, R., Shepherd, R., Downing, A. and Lawson, K. (2016). The perspectives of obese women receiving antenatal care: A qualitative study of women's experiences. Women and Birth, 29(2), pp.189-195.

Lavender, T. and Smith, D. (2015). Seeing it through their eyes: a qualitative study of the pregnancy experiences of women with a body mass index of 30 or more. Health Expectations, 19(2), pp.222-233.

Seneviratne, S., Jiang, Y., Derraik, J., McCowan, L., Parry, G., Biggs, J., Craigie, S., Gusso, S., Peres, G., Rodrigues, R., Ekeroma, A., Cutfield, W. and Hofmana, P. (2017). Effects of Antenatal Exercise in Overweight and Obese Pregnant Women on Maternal and Perinatal Outcomes. Obstetric Anesthesia Digest, 37(1), pp.34-35.

(22) McDonald, S., Viaje, K., Rooney, R., Jarde, A., Giglia, L., Maxwell, C., Small, D., Pearce Kelly, T., Sabatino, L. and Thabane, L. (2018). A clinical carepath for obese pregnant women: a pragmatic pilot cluster randomized controlled trial. The Journal of Maternal-Fetal \& Neonatal Medicine, pp.1-8.

(23) Timur, B., Timur, H., Tokmak, A., Isik, H. and Eyi, E. (2018). The Influence of Maternal Obesity on Pregnancy Complications and Neonatal Outcomes in Diabetic and Nondiabetic Women. Geburtshilfe und Frauenheilkunde, 78(04), pp.400-406.

Wang, C., Wei, Y., Zhang, X., Zhang, Y., Xu, Q., Sun, Y., Su, S., Zhang, L., Liu, C., Feng, Y., Shou, C., Guelfi, K., Newnham, J. and Yang, H. (2017). A randomized clinical trial of exercise during pregnancy to prevent gestational diabetes mellitus and improve pregnancy outcome in overweight and obese pregnant women. American Journal of Obstetrics and Gynecology, 216(4), pp.340-351.

(25) Vesco, K., Karanja, N., King, J., Gillman, M., Leo, M., Perrin, N., McEvoy, C., Eckhardt, C., Smith, K. and Stevens, V. (2014). Efficacy of a group-based dietary intervention for limiting gestational weight gain among obese women: A randomized trial. Obesity, 22(9), pp.1989-1996.

Hui, A., Back, L., Ludwig, S., Gardiner, P., Sevenhuysen, G., Dean, H., Sellers, E., McGavock, J., Morris, M., Jiang, D. and Shen, G. (2014). Effects of lifestyle intervention on dietary intake, physical activity level, and gestational weight gain in pregnant women with different pre-pregnancy Body Mass Index in a randomized control trial. BMC Pregnancy and Childbirth, 14(1). 
Gesche, J., Renault, K., Nørgaard, K. and Nilas, L. (2014). Representativeness of Participants in a Lifestyle Intervention Study in Obese Pregnant Women - the Difference between Study Participants and Non-Participants. Obesity Facts, 7(6), pp.351-360.

(28) Hayes, L., Bell, R., Robson, S. and Poston, L. (2014). Association between Physical Activity in Obese Pregnant Women and Pregnancy Outcomes: The UPBEAT Pilot Study. Annals of Nutrition and Metabolism, 64(3-4), pp.239-246.

(29) Smith, D., Cooke, A. and Lavender, T. (2012). Maternal obesity is the new challenge; a qualitative study of health professionals' views towards suitable care for pregnant women with a Body Mass Index (BMI) $\geq 30 \mathrm{~kg} / \mathrm{m} 2$. BMC Pregnancy and Childbirth, 12(1).

(30) Charnley, M., Coufopoulos, A., Weeks, A. and Abayomi, J. (2016). A qualitative exploration of the lived experiences of pregnant women with BMI $\geqslant 35 \mathrm{~kg} / \mathrm{m} 2$. Proceedings of the Nutrition Society, 75(OCE3).

(31) Mandel, D. and DeJoy, S. (2016). A Qualitative Study of the Maternity Care Experiences of Women With Obesity. Journal of Obstetric, Gynecologic \& Neonatal Nursing, 45(3), p.S48. 